Meta

Journal des traducteurs

Translators' Journal

\title{
Pour une contribution sémiotique de la traduction et de la terminologie
}

\section{Raymond Tourangeau}

Volume 25, numéro 3, septembre 1980

URI : https://id.erudit.org/iderudit/004498ar

DOI : https://doi.org/10.7202/004498ar

Aller au sommaire du numéro

Éditeur(s)

Les Presses de l'Université de Montréal

ISSN

0026-0452 (imprimé)

1492-1421 (numérique)

Découvrir la revue

Citer cet article

Tourangeau, R. (1980). Pour une contribution sémiotique de la traduction et de la terminologie. Meta, 25(3), 310-315. https://doi.org/10.7202/004498ar d'utilisation que vous pouvez consulter en ligne.

https://apropos.erudit.org/fr/usagers/politique-dutilisation/ 


\title{
Pour une contribution sémiotique de la traduction et de la terminologie
}

RAYMOND TOURANGEAU

\begin{abstract}
«Ce qu'il nous faut deviner est caché derrière les mots et les actes, caché derrière les cuures. II nous faut revenir au point de départ pour connaitre le lieu de l'arrivée. Entre la réalité brute, et la reconquête de la réalité pure, il y a tout le voyage du verbe. Mais puisque notre connaissance ne se parachève que par le langage, nous n'avons pas le choix. Il nous faut rattraper la vérité dans le mensonge, retrouver l'impulsion dans le mouvement.»
\end{abstract}

Jean-Marie Le Clézio,

L'Extase matérielle, Paris, Gallimard, 1967, «Idées $» n^{\circ} 239$, p. 171.

\section{INTRODUCTION}

Ce travail présente, dans sa première partie, un apport possible de la traduction à une sémiotique des textes écrits, génératrice d'une discussion sur les divers types de productions textuelles et, notamment, sur les notions de discours, de messages et de langages; il milite encore, en deuxième partie, pour une contribution sociolinguistique et sémiotique de la terminologie, qui permettrait de jeter un nouvel éclairage sur les sous-codes de langages de spécialité.

En contrepoint, il s'agit de faire voir que, par-delà la médiation qu'elles opèrent au sein du système général de la communication, par-delà l'archicompétence cultivée qu'elles exigent du praticien, les disciplines traduisante et terminographique apparaissent comme des activités sémiotiques, de véritables cheminements vers la culture (lato sensu).

\section{Traduction et SÉMIOTIQUe TEXTUELLE*}

De la traduction à la sémiotique textuelle

La traduction, dit-on, mène à tout. En effet, «condamné» par la nature même de son travail à la généralité, le traducteur est amené à exercer son activité sur des textes relatifs aux domaines les plus représentatifs de la culture dans laquelle il se situe. Domaines commercial et économique, technique et scientifique, médical et pharmaceutique, juridique et littéraire, etc., dictés, en

* Le terme de «sémiotique textuelle * signifie ici «sémiotique des textes écrits "; il ne désigne aucune théorie littéraire ayant cours. 
amont, par les priorités de notre ordre culturel et attestés, en aval, par les programmes universitaires d'études en traduction.

Placé au cœur de la production textuelle privilégiée (puisqu'elle vaut la peine d'être écrite, puis traduite en vue d'une plus grande diffusion) de la synchronie culturelle dont il fait partie, le traducteur, qui opère une médiation herméneutique et un transfert interlinguistique entre destinateur et destinataire, prend part active au procès de la signification et mérite voix (si discrète soitelle) au chapitre d'une sémiotique textuelle.

Mais d'où une telle théorie tire-t-elle son importance? D'abord, le texte écrit, fixé pour fins de conservation et d'économie, reçoit implicitement caution culturelle; ce sera d'autant plus le cas du texte destiné à la traduction. Une sémiotique textuelle répond encore au besoin, pressenti par Todorov, d'une science générale des discours, dont la poétique, dans son rôle «éminemment transitoire», constituerait le prologue (voir Poétique, p. 108-109). Puis, une fois le débat porté sur le terrain de la sémiologie, qui considère également «les innombrables variétés de langages à travers lesquels une culture se constitue» (selon le mot de M. Eco), il devient impossible, par souci de scientificité, de privilégier a priori une production (fût-elle littéraire et, partant, investie d'un prestige transhistorique) au détriment des autres.

\section{Discours, messages, langages}

En vue d'une sémiotique textuelle, il convient de préciser la notion de discours, dans une perspective large et synchronique, à la mesure des différentes productions écrites de notre époque.

Le mot de «discours», dans son acception scientifique, linguistique, (voir Lexis, p. 533) réfère au «langage mis en action et assumé par le sujet parlant», donc à la concaténation, qui fait partie intégrante de l'acte de parole - et d'écriture. La notion de discours implique dès lors nécessairement l'action volontaire d'un destinateur qui construit (procède au choix des mots et à leur enchaînement), puis produit (actualise, verbalise, écrit) son message. Ainsi, des diverses productions textuelles, seules celles qui s'intègrent au domaine littéraire répondent en tout point aux critères du discours du fait qu'elles résultent de l'acte de volonté (qui seulement ici peut être appelé "création») d'un destinateur particulièrement individualisé (qui, seul, peut être appelé "auteur» ou «écrivain»). A ce point individualisé, en fait, que l'auteur et son œuvre ne font qu'un dans l'esprit du destinataire. Aussi peut-on demander à des consommateurs de produits littéraires de quoi ils ont rassasié leur appétit récemment et s'entendre répondre: «J'ai lu du Giono», «J'ai lu Hugo», «Méthodes de Ponge», etc. Selon toute vraisemblance, personne ne répondra: « $J$ 'ai lu de la littérature», tant il est vrai que le produit littérature n'en est pas un comme les autres, qui se laisserait apercevoir (je ne dis pas réduire à son entité théorique) hors des cuvres qui le constituent, indépendamment d'un «je»pleinement assumé par l'auteur. Celui-ci met en forme un message, le met en oeuvre (car seul ce message au deuxième degré constitue une «œuvre», qui, si elle s'intègre au système général de la communication, se distingue par sa fonction poétique, par 
sa visée esthétique et par son caractère non informatif). Ainsi, les fautes de sens les plus fréquentes en traduction littéraire sont dites «erreurs d'interprétation» (d'infidélité au «je » de l'auteur) par opposition aux diverses atteintes au contenu informatif des messages commerciaux ou techniques, par exemple. Encore une fois, donc, la question de la fonction référentielle, essentielle à toute production textuelle, littérature exceptée, fait surface. C'est dire que le message a généralement pour but premier de renseigner, de renvoyer à une réalité extralinguistique, tandis que le texte littéraire met l'accent sur les fonctions poétique (forme du message) et expressive (du fait que, dans notre synchronie culturelle, le $\ll j \mathrm{je}$ de l'auteur demeure indissociable de son œuvre).

Il me semble donc que, dans une perspective d'ensemble, les productions autres que les ouvres littéraires ne constituent pas des discours mais des messages formés à partir de «langages» (économique, juridique, etc.), de manières de parler propres à des groupes partageant une même expérience (extralinguistique) et organisant autour de celle-ci des champs lexicaux (ou terminologiques, suivant l'optique) qui lui soient adéquats, des terminologies fort souvent étanches à la langue commune. Les termes, pour remplir leur fonction référentielle première, sont le plus possible univoques, d'une impérative précision, alors que, comme l'a vu Chklovski, le discours littéraire, sans doute à cause de l'absence de référent et de l'emploi qu'il fait des mots, polysémiques, de la langue commune, se distingue par son ambiguité.

J'estime encore qu'il n'existe d'œuvre littéraire que si le choix du locuteur est illimité, que si l'écrivain s'accorde toute liberté de concaténation à l'intérieur des virtualités de la langue, voire dans les diverses terminologies, se réservant le loisir de décloisonner les champs de l'expérience et, partant, les champs lexicaux qui leur sont associés, de transgresser les normes pour créer un discours proprement expressif, original, qui se donne à saisir plus qu'il ne donne à comprendre.

$\mathrm{Si}$, à ce stade, l'on reprenait notre enquête, cette fois auprès de «scientifiques», des réponses comme: «J'ai lu mes maths» ou «J'ai lu un traité de physique», sans mention d'auteur, auraient sûrement une certaine récurrence. Les productions textuelles techniques, d'ailleurs le plus souvent aux mains du grand capital et des ministères et sociétés d'État, sont généralement anonymes (quel ingénieur a donc écrit le guide d'entretien de votre voiture?) et remplissent d'abord fonction référentielle. Le traducteur de ces textes, qui partage l'«invisibilité» de leur rédacteur (respectant, avec Barthes, la distinction écrivant-écrivain, ne parlons plus ici d'auteur), doit s'en tenir au degré zéro du style et à la terminologie propre au domaine. Et ce même traducteur, qui travaille journellement sur ces textes, demande qu'on imagine seulement le pseudodiscours médical coupé de sa terminologie...

Par ailleurs, les textes scientifiques de chercheurs comme Selye, Samuelson, Jakobson, etc. s'inscrivent, dans leurs domaines respectifs, dans ce que les Anglo-Saxons appellent «the Great Conversation » (discours de la Connaissance) et demeurent inséparables du nom de leur destinateur. D'emblée, ils sont classés dans la catégorie du discours. On reconnaîtra toutefois que les éléments lexicaux 
actualisés dans ce type de discours se rattachent à une terminologie ou à une métalangue similaire à celles des diverses productions précédemment identifiées comme des «langages». Ce qui me ramène à mon assertion ci-dessus selon laquelle «de toutes les productions textuelles, seules celles qui s'intègrent au domaine littéraire répondent en tout point aux critères du discours", c'està-dire que la littérature est discours par essence et que tout «langage» peut incidemment, dans certaines manifestations individuelles, donner lieu à des discours.

\section{POUR UNE CONTRIBUTION SOCIOLINGUisTIQUE ET SÉMIOTIQUE DE LA TTERMINOLOGIE: LE DESSOUS DES FICHES}

La traduction et la terminologie, activités pratiques, répondent respectivement aux besoins d'intercompréhension et d'uniformisation, voire de normalisation, inhérents à la situation de diglossie qui justifie jusqu'à leur existence.

La terminologie, qui de plus en plus parvient à se démarquer de la tradition lexicographique, garde partie intimement liée avec la pratique traduisante, qu'elle s'emploie à rationaliser, en recherche ponctuelle et thématique, à coups de fiches et de lexiques. Toutefois, si elle s'inscrit, pour ainsi dire, dans la foulée de la traduction, la terminologie, activité onomasiologique, d'encodage, revêt encore une portée sociolinguistique en ce qu'elle reflète, en amont, des usages propres à certains groupes sociaux nettement identifiables et en ce qu'elle produit, en aval, des lexiques faisant autorité ou souvent perçus comme tel.

C'est dire que la terminologie enregistre les usages en situation, maxi(contexte socioculturel qui conditionne usages et usagers) et mini-situation (contexte linguistique au stade de la rédaction de la fiche ou du lexique), qu'elle évolue, donc, d'un champ situationnel (au moment de la collecte d'information) à un champ conceptuel (propre aux usagers de la terminologie particulière) vers, enfin, un champ terminologique (présenté sous forme de fiches, de schémas ou de lexiques). Aussi le point de départ de sa démarche, ses orientations référentielle et situationnelle fondamentales et, partant, sa prise directe sur le vécu sociolinguistique, devraient-elles, à terme, permettre l'extension de la stricte terminographie en une véritable terminologie (suivant le couple oppositif suggéré par Alain Rey), discipline théorique à part entière, dont la contribution sémiotique à la conversation, déjà résolument engagée, sur le procès de la signification et de la culture justifie qu'on fonde en elle des espoirs sérieux.

Dès lors, la terminologie se prolonge d'une praxéologie qui se fonde moins sur des critères a priori de scientificité que sur les résultats terminaux qu'elle permet d'obtenir. Ceux-ci, les différentes terminologies, peuvent être regardés comme pratiques signifiantes particulières, fonctionnelles tant au niveau linguistique que social, comme modes de signification et de production. L'unité terminologique, ci-devant clé pour la traduction et élément fonctionnel au sein d'un langage de spécialité et, par incidence, du système général de la langue, fait encore office d'unité fonctionnelle dans le système de production social et culturel. 
Cette approche, conforme à la démarche terminologique et à la réflexion linguistique de Meillet, de Wittgenstein et, plus près de nous, de Ladmiral, se fonde sur la reconnaissance, quotidienne chez le traducteur, que les mots ont des emplois plutôt que des sens (ou, de façon moins «extrême», qu'ils ne reçoivent, de façon plus ou moins motivée, de sens qu'à l'usage, c'est-à-dire en maxi- et en mini-situation). Dans cette optique, les unités terminologiques s'intègrent donc au progrès linguistique comme unités distinctives actualisées dans des messages ou discours renvoyant à leurs archétypes fichés, eux-mêmes fonctionnels à l'intérieur du sous-code d'un langage de spécialité et, éventuellement, du code de la langue, au procès sociolinguistique comme unités fonctionnelles répondant aux besoins de communication particuliers d'un groupe social ou socioprofessionnel, de même qu'au procès sémiotique comme unités fonctionnelles au sein des divers modes de signification et de production de notre synchronie culturelle.

Il importe ainsi de mettre en évidence l'étroite connexité qui unit dans l'osmose culturelle champs terminologiques et champs d'activité, productions langagières et pratiques sociales. De toutes les disciplines linguistiques, la terminologie est à cet égard la mieux placée pour éclairer les phénomènes parallèles, décriés par d'aucuns, de babélisation, de surspécialisation et de ramification de la culture, lot néanmoins «normal» d'une civilisation qui fonde ses structures linguistiques et sociales sur l'arbitraire, d'une part, et sur la multiplicité de l'expérience, d'autre part.

La terminologie, discipline-charnière, est donc à même de bien faire voir combien les pratiques signifiantes et culturelles participent d'une même conjoncture qui autorise leur éclatement sans toutefois jamais permettre leur cloisonnement définitif par le recours nécessaire, l'intégration obligée au véhicule privilégié de notre culture: le système de la langue.

\section{Conclusion}

Ainsi, pour porter un coup d'arrêt à la perception, encore trop répandue, de la traduction et de la terminologie comme pratiques serviles ne tirant leur raison d'être que d'une contingence de diglossie, il importe de susciter chez les praticiens intéressés une relativisation de leur action, en définitive toujours fort «ponctuelle», dans la perspective, globale, sémiologique, du fonctionnement des pratiques symbolisantes au sein de la vie sociale.

À condition d'éviter le piège des extrêmes, celui du cloisonnement des disciplines, qui pourrait prendre la forme d'une traduction «aterminologique» ou celle d'une terminologie tenue pour l'auxiliaire ultime de la traduction, et celui d'une perspective par trop "globalisante», qui perdrait de vue les conditions d'exercice de la traduction et de la terminologie, et à condition de rattacher l'étude de ces deux disciplines au cadre sémiologique (que je tiens pour le seul à pouvoir rendre compte du phénomène, central, de la production de sens), il deviendra possible pour le praticien de passer de l'état d'《actant» anonyme dans une trame tissée de l'extérieur à celui d'acteur consciemment engagé dans le procès, trop humain pour lui être étranger, de la signification et de la culture. 


\section{BIBLIOGRAPHIE}

BAR'THES, Roland, Le Degré zéro de l'écriture, suivi des Éléments de sémiologie, Paris, Gonthier, 1965 , coll. Médiations $\mathrm{n}^{\circ} 40$.

CHKLOVSKI, V., L'Art comme procédé, in Théorie de la littérature, textes des Formalistes russes réunis, présentés et traduits par Tzvetan Todorov, Paris, Éd. du Seuil, 1965.

DUBOIS, Jean et collaborateurs, Lexis, dictionnaire de la langue française, Paris, Larousse, 1975.

DUBUC, Robert, Manuel pratique de terminologie, Linguatech, Montréal, Conseil international de la langue française, Paris, 1978.

DUCROT, Oswald et Tzvetan TODOROV, Dictionnaire encyclopédique des sciences du langage, Paris, Éd. du Seuil, 1972.

JAKOBSON, Roman, Essais de linguistique générale, Paris, Éd. de Minuit, 1963, coll. Points $n^{\circ} 17$.

LADMIRAL, Jean-René, Traduire: théorèmes pour la traduction, Paris, Petite Bibliothèque Payot $\mathrm{n}^{\circ} 366,1979$.

RÉGIE DE LA LANGUE FRANÇAISE, Essai de définition de la terminologie, actes du colloque international de terminologie, Québec, Manoir du Lac Delage, oct. 1975, Éd. officiel du Québec, 1976.

TODOROV, Tzvetan, Poétique, in Qu'est-ce que le structuralisme?, Paris, Ed. du Seuil, 1968, coll. Points $n^{\circ} 45$. 